\title{
AVALIAÇÃO DA EFICIÊNCIA DE DELTAMETRINA (K-OTHRINE 50FW), EM DOSES BAIXAS, NO CONTROLE DE TRIATOMÍNEOS EM SÃO SEBASTIÃO DO UMBUZEIRO, PARAÍBA
}

\author{
Carlos Brisola Marcondes ${ }^{1}$ e Cláudio Teixeira Pinto2
}

\begin{abstract}
Testamos a deltametrina ( $K$-Othrine $50 F W$ ) no controle de triatomíneos em área de semi-árido, com cerca de $56,6 \%$ de positividade inicial no intradomicílio. Usamos, em 402 Unidades Domiciliares, 125, 75, 60 ou 50ml por bomba de 10 litros, equivalendo, se aplicados em $250 \mathrm{~m}^{2}$, a $25,15,12 \mathrm{ou} 10 \mathrm{mg} / \mathrm{m}^{2}$. Os índices de invasão, colonização e cumulativo no intradomicilio e de positividade no peridomicílio foram muito baixos e similares nos quatro grupos, nos 15 meses de avaliação, indicando poderem as doses menores substituir a de $25 \mathrm{mg} / \mathrm{m}^{2}$. Houve predominância de $T$. infestans, seguido de T. pseudomaculata, nas casas, e este predominou no peridomicílio, após a pulverização.
\end{abstract}

Palavras-chaves: Deltametrina. Piretróide. Doença de Chagas. Semi-árido. Triatominae.

A deltametrina vem sendo testada, há vários anos, para o controle de triatomíneos, tendo sido obtida proteção por cerca de um ano, com doses de $80 \mathrm{a}$ $200 \mathrm{mg} / \mathrm{m}^{2} 711$, com melhores resultados com a formulação $\mathrm{FW}^{7}$. Dias e $\mathrm{col}^{3}$ evidenciaram boa proteção com $25 \mathrm{mg} / \mathrm{m}^{2}$, concentração utilizada pela SUCAM, em formulação $\mathrm{FW}$, em vários estados do Brasil.

No presente trabalho, relatamos os resultados de testes com a dose de $25 \mathrm{mg} / \mathrm{m}^{2}$ e menores, com o objetivo de verificar se estas poderiam substituir, com economia, aquela dose.

\section{MATERIAL E MÉTODOS}

O trabalho foi desenvolvido no município de São Sebastião do Umbuzeiro. Este fica na microrregião de Cariris Velhos, no extremo sul da Paraíba, com pluviosidade anual média de $600 \mathrm{~mm}$, temperaturas máximas e mínimas mensais variando, respectivamente, entre 28 e $33^{\circ} \mathrm{C}$ e 14 e $20^{\circ} \mathrm{C}$ e está na categoria Bswh' de Koeppen 12 .

Ocorriam na área Triatoma infestans, T. brasiliensis, T. pseudomaculata, Panstrongylus megistus e $P$. lutzi, com base em Levantamento Triatomínico (L.T.) da SUCAM, feito em 1983. A partir

\footnotetext{
${ }^{1}$ Departamento de Fisiologia e Patologia/Núcleo de Medicina Tropical, Centro de Ciências da Saúde, Universidade Fe deral da Paraiba, João Pessoa, PB. ${ }^{2}$ Quimio Produtos Químicos Comércio e Indústria S.A. Rio de Janeiro, RJ.

Endereço para correspodência: Prof. Carlos Brisola Marcondes. Depto. de Fisiologia e Patologia/UFPB. Campus Universitário. 58000 - Joào Pessoa, PB
}

Recebido para publicação em 26/12/88. deste L.T., escolhemos as 23 localidades de maior positividade no município, situadas no norte deste. A positividade nestas localidades foi de 307 de 542 casas $(56,6 \%)$ e 334 de 542 Unidades Domiciliares (U. Ds.) $(61,6 \%)$. Um L.T. realizado no municipio de Camalaú, próximo da área deste estudo, em 1987, indicou positividade de $60,4 \%$ das U. Ds. que, somando-se mais 24 U. Ds. positivas na pulverização, passou para $69,6 \%$, bem maior que no L. T. de 1983 , com $49,8 \%$. Houve aumento na proporção de U.Ds. com $T$. infestans de $21,0 \%$ para $31,1 \%$ (C. B. Marcondes \& L. R. Benvenutti: dados não publicados). Sendo as duas áreas próximas e semelhantes, é provável que a positividade da área do teste fosse, em 1986, igual ou maior que a de 1983. A população foi instruída a coletar e preservar, em sacos plásticos, os insetos mortos na pulverização, mas o material sofreu rápida deterioração e não pôde ser analisado.

As U. Ds. foram distribuidas, de modo aleatório, em quatro grupos, com base nos mapas da SUCAM, incluindo casas adjacentes num mesmo grupo.

Uma equipe da SUCAM, constituída de 25 funcionários, 20 dos quais aplicando inseticida, pulverizou 402 U. Ds., em julho de 1986. Foi usado, nos grupos A (116 U. Ds.), B (89 U. Ds.), C (96 U. Ds. e D (101 U. Ds.), K-Othrine FW 5\%, respectivamente, nas diluições de $125,75,60$ e $50 \mathrm{ml}$ por bomba de 10 litros de água. Os guardas, de acordo com a metodologia usual da SUCAM, aplicaram o material nas paredes internas das casas até $3 \mathrm{~m}$ de altura e, no caso destas serem baixas, também em parte do teto, além dos beirais, objetos domésticos sujeitos a dar abrigo a triatomíneos e possiveis criadouros peridomiciliares. Como o pessoal trabalhou na velocidade habitual da SUCAM e o material que sobrava de uma bomba, apos tratar uma U. D., era usado na seguinte do mesmo 
Marcondes CB, Pinto CT. Avaliação da eficiência de deltametrina (K-Othrine 50FW), em doses baixas, no controle de triatomíneos em São Sebastião do Umbuzeiro, Paraíba. Revista da Sociedade Brasileira de Medicina Tropical 22: 85-90. Abr-Jun. 1989

grupo, é provável que o líquido de cada bomba tenha sido aplicado em cerca de $250 \mathrm{~m}^{2}$ de parede. Neste caso, as doses prováveis foram de $25,15,12 \mathrm{e} 10 \mathrm{mg} \mathrm{de}$ ingrediente ativo $/ \mathrm{m}^{2}$, respectivamente, para os grupos A, B, C e D. Não medimos as casas nem a quantidade de material gasto em cada uma, devido a dificuldades operacionais e à provável influência da quantidade e altura das paredes, notada também por Oliveira Filho e col.

Equipes de pesquisa, constituidas de 22 a 25 guardas capturadores, sob supervisão de inspetores da SUCAM e de um dos Autores (CMB), obtinham amostras de insetos nas U. Ds. existentes e acessíveis nas 23 localidades, em 9 avaliações $(1,2,3,6,8,10$, 12,14 e 15 meses). Os guardas faziam captura manual de triatomíneos vivos, usando piretro a $2 \%$ como desalojante em frestas profundas de paredes e outros abrigos de difícil visualização. Os componentes das equipes variavam e eram escolhidos por sua maior experiência e disponibilidade na época de cada avaliação.

A partir do material capturado no intradomicilio, em proporção ao número de U. Ds. pesquisadas, calculamos dois índices: a) de invasão-encontro ex- clusivo de adultos; b) de colonização-encontro de ninfas. Usando a quantidade de U. Ds. com insetos na avaliação em análise e/ou em anterior, calculamos o índice cumulativo; para este cálculo, incluímos as $\mathbf{U}$. Ds. não pesquisădas, desde que tivessem sido positivas em avaliação anterior. Com base no material do peridomicilio, calculamos um indice de positividade deste, constituido de: U. Ds. nas quais foram obtidos insetos no peridomicílio/U. Ds. pesquisadas. Este índice, para o intradomicilio, pode ser calculado pela soma dos índices de invasão e de colonização. Os índices dos grupos foram comparados, para cada avaliação, por meio do teste de $\mathrm{X}^{2}$, ao nível de $5 \%$.

\section{RESULTADOS}

Houve grande mortalidade inicial de triatomíneos e permanência prolongada dos efeitos, com alguns casos de irritação leve e passageira de pele e mucosas em pessoal da SUCAM. Nas primeiras avaliações, observamos grande quantidade de tritomíneos mortos, principalmente $T$. infestans, e cascas de ovos em frestas de paredes.

A Tabela 1 mostra os indices de invasão, a Tabela 2 os de colonização e a Tabela 3 os cumula-

Tabela 1 - Quantidades de Unidades Domiciliares só com adultos/quantidades examinadas em cada avaliação e indice de invasão.

\begin{tabular}{ccccccccccc}
\hline Grupos & \multicolumn{10}{c}{ Avaliações } \\
\hline & $1 \mathrm{a}$ & $2 \mathrm{a}$ & $3 \mathrm{a}$ & $4 \mathrm{a}$ & $5 \mathrm{a}$ & $6 \mathrm{a}$ & $7 \mathrm{a}$ & $8 \mathrm{a}$ & $9 \mathrm{a}$ \\
\hline $\mathrm{A}$ & $0 / 103$ & $2 / 105$ & $2 / 100$ & $1 / 105$ & $1 / 93$ & $1 / 97$ & $0 / 94$ & $2 / 93$ & $1 / 98$ \\
& $(0,0)$ & $(1,9)$ & $(2,0)$ & $(1,0)$ & $(1,1)$ & $(1,0)$ & $(0,0)$ & $(2,2)$ & $(1,0)$ \\
$\mathrm{B}$ & $0 / 83$ & $0 / 80$ & $0 / 80$ & $1 / 76$ & $0 / 82$ & $1 / 73$ & $0 / 78$ & $1 / 80$ & $0 / 76$ \\
& $(0,0)$ & $(0,0)$ & $(0,0)$ & $(1,3)$ & $(0,0$ & $(1,4)$ & $(0,0)$ & $(1,2)$ & $(0,0)$ \\
$\mathrm{C}$ & $1 / 82$ & $2 / 85$ & $0 / 85$ & $1 / 77$ & $0 / 77$ & $0 / 71$ & $0 / 80$ & $1 / 77$ & $1 / 74$ \\
& $(1,2)$ & $(2,4)$ & $(0,0)$ & $(1,3)$ & $(0,0)$ & $(0,0)$ & $(0,0)$ & $(1,3)$ & $(1,4)$ \\
$\mathrm{D}$ & $0 / 85$ & $0 / 83$ & $0 / 87$ & $2 / 80$ & $0 / 80$ & $2 / 82$ & $0 / 84$ & $2 / 80$ & $0 / 78$ \\
& $(0,0)$ & $(0,0)$ & $(0,0)$ & $(2,5)$ & $(0,0)$ & $(2,4)$ & $(0,0)$ & $(2,5)$ & $(0,0)$ \\
\hline Sign. & NS & NS & NS & NS & NS & NS & NS & NS & NS \\
\hline
\end{tabular}

Tabela 2 - Quantidades de Unidades Domiciliares com ninfas no intradomicílio/quantidades examinadas em cada avaliação e indices de colonização.

\begin{tabular}{cccccccccc}
\hline Grupos & \multicolumn{10}{c}{ Avaliações } \\
\hline & $1 \mathrm{a}$ & $2 \mathrm{a}$ & $3 \mathrm{a}$ & $4 \mathrm{a}$ & $5 \mathrm{a}$ & $6 \mathrm{a}^{\mathrm{a}}$ & $7 \mathrm{a}$ & $8 \mathrm{a}$ & $9 \mathrm{a}$ \\
\hline $\mathrm{A}$ & $0 / 103$ & $2 / 105$ & $1 / 100$ & $0 / 105$ & $0 / 93$ & $1 / 97$ & $0 / 94$ & $0 / 93$ & $1 / 98$ \\
& $(0,0)$ & $(1,9)$ & $(1,0)$ & $(0,0)$ & $(0,0)$ & $(1,0)$ & $(0,0)$ & $(0,0)$ & $(1,0)$ \\
$\mathrm{B}$ & $1 / 83$ & $2 / 80$ & $1 / 80$ & $0 / 76$ & $1 / 82$ & $0 / 73$ & $0 / 78$ & $0 / 80$ & $0 / 76$ \\
& $(1,2)$ & $(2,5)$ & $(1,2)$ & $(0,0)$ & $(1,2)$ & $(0,0)$ & $(0,0)$ & $(0,0)$ & $(0,0)$ \\
$\mathrm{C}$ & $3 / 82$ & $3 / 85$ & $1 / 85$ & $0 / 77$ & $2 / 77$ & $0 / 71$ & $1 / 80$ & $0 / 77$ & $0 / 74$ \\
& $(3,7)$ & $(3,5)$ & $(1,2)$ & $(0,0)$ & $(2,6)$ & $(0,0)$ & $(1,2)$ & $(0,0)$ & $(0,0)$ \\
$\mathrm{D}$ & $0 / 85$ & $1 / 83$ & $1 / 87$ & $0 / 80$ & $1 / 80$ & $1 / 82$ & $2 / 84$ & $2 / 80$ & $1 / 78$ \\
& $(0,0)$ & $(1,2)$ & $(1,2)$ & $(0,0)$ & $(1,2)$ & $(1,2)$ & $(2,4)$ & $(2,5)$ & $(1,3)$ \\
\hline Sign. & NS & NS & NS & NS & NS & NS & NS & NS & NS \\
\hline
\end{tabular}


Marcondes CB, Pinto CT. Avaliafāo da eficiência de deltametrina (K-Othrine 50FW), em doses baixas, no controle de triatomineos em São Sebastião do Umbuzeiro, Paraiba. Revista da Sociedade Brasileira de Medicina Tropical 22: 85-90, Abr-Jun, 1989

Tabela 3 - Quantidades de Unidades Domiciliares positivas para triatomíneos no intradomicílio na avaliação em análise e/ou em anterior/quantidades examinadas em cada avaliação + positivas em avaliação anterior e não avaliadas (fechadas) e índices cumulativos.

\begin{tabular}{cccccccccc}
\hline Grupos & & \multicolumn{10}{c}{ Avaliações } \\
\hline & $1 \mathrm{a}$ & $2 ! \mathrm{a}$ & $3 \mathrm{a}$ & $4 \mathrm{a}$ & $5 \mathrm{a}$ & $6 \mathrm{a}$ & $7 \stackrel{\mathrm{a}}{8}$ & $8 \mathrm{a}$ & $9 \mathrm{a}$ \\
\hline $\mathrm{A}$ & $0 / 103$ & $4 / 105$ & $5 / 100$ & $9 / 106$ & $10 / 94$ & $11 / 98$ & $11 / 104$ & $15 / 96$ & $15 / 100$ \\
& $(0,0)$ & $(3,8)$ & $(5,0)$ & $(8,5)$ & $(10,6)$ & $(11,2)$ & $(10,6)$ & $(15,6)$ & $(15,0)$ \\
$\mathrm{B}$ & $0 / 83$ & $2 / 80$ & $3 / 80$ & $3 / 76$ & $4 / 82$ & $5 / 73$ & $5 / 78$ & $6 / 80$ & $6 / 76$ \\
& $(1,2)$ & $(2,5)$ & $(3,8)$ & $(4,0)$ & $(4,9)$ & $(6,8)$ & $(6,4)$ & $(7,5)$ & $(7,9)$ \\
$\mathrm{C}$ & $4 / 82$ & $7 / 85$ & $7 / 88$ & $7 / 78$ & $7 / 77$ & $7 / 72$ & $7 / 80$ & $7 / 78$ & $8 / 76$ \\
& $(4,9)$ & $(8,2)$ & $(8,0)$ & $(9,0)$ & $(9,1)$ & $(9,7)$ & $(8,8)$ & $(9,0)$ & $(10,5)$ \\
$\mathrm{D}$ & $0 / 85$ & $1 / 83$ & $1 / 87$ & $3 / 80$ & $3 / 80$ & $5 / 82$ & $6 / 84$ & $7 / 80$ & $7 / 78$ \\
& $(0,0)$ & $(1,2)$ & $(1,2)$ & $(3,8)$ & $(3,8)$ & $(6,1)$ & $(7,1)$ & $(8,8)$ & $(9,0)$ \\
\hline Sign. & S & NS & NS & NS & NS & NS & NS & NS & NS \\
\hline
\end{tabular}

tivos. Os indices foram muito baixos na maior parte do periodo, havendo redução nos dois primeiros, nos grupos A, B e C, após as duas primeiras avaliações, possivelmente devido à eliminação dos remanescentes das populações iniciais, com o contato com as superficies tratadas. Os índices cumulativos foram bem maiores, devido à pouca repetição de encontro de insetos nas mesmas U. Ds. Apesar dos adultos não serem os únicos invasores possíveis, são os mais prováveis e sua presença exclusiva em certas amostras deve ser ressaltada, por terem sido obtidas pelo pessoal da SUCAM e não pela população, o que indica algum contato com superficies tratadas mas não o estabelecimento de colônias.

Com uma exceção, no indice cumulativo, nenhuma das diferenças foi significativa. $O$ índice de positividade no intradomicilio, não mostrado em tabela, por sua fácil obtenção, também só teve uma diferença significativa, na primeira avaliação. Isto indica a ausência de diferenças significativas entre os efeitos das doses do inseticida.

Das 3.043 amostras obtidas, 54 foram positivas no intradomicilio. As quantidades de amostras positivas para cada espécie são mostradas na Tabela 4,

Tabela 4 - Quantidades de amostras positivas no intradomicilio para cada espécie, nos grupos A, B, C e D, após a pulverização.

\begin{tabular}{lccccc}
\hline \multicolumn{1}{c}{ Espécies } & \multicolumn{5}{c}{ Grupos } \\
\hline & A & B & C & D & Total \\
\hline T. infestans & 4 & 3 & 9 & 10 & 26 \\
T. pseudomaculata & 5 & 3 & 4 & 5 & 17 \\
T. brasiliensis & 5 & 0 & 1 & 0 & 6 \\
$P$. megistus & 1 & 2 & 2 & 0 & 5 \\
$P$. lutzi & 0 & 0 & 0 & 0 & 0 \\
\hline Total & 15 & 8 & 16 & 15 & 54 \\
\hline
\end{tabular}

totalizándo 55 amostras, devido à ocorrência de uma com $T$. infestans e $T$. pseudomaculata juntos. Podemos notar a maior quantidade de amostras positivas para $T$. infestans, seguida de $T$. pseudomaculata, indicando, provavelmente, maior freqüência destas espécies nas casas e/ou maior capacidade de desenvolvimento de populações intradomiciliares, por proliferação de insetos residuais ou reinvasão.
Os indices de positividade no peridomicilio (Tabela 5) foram muito baixos no período estudado, sem diferenças significativas, com exceção da sétima e da oitava avaliação, o que indica que, também neste ambiente, as doses tiveram efeitos similares. 
Marcondes CB, Pinto CT. Avaliação da eficiència de deltametrina ( $K$-Othrine 50FW), em doses baixas, no controle de triatomíneos em São Sebastião do Umbuzeiro, Paraiba. Revista da Sociedade Brasileira de Medicina Tropical 22: 85-90, Abr-Jun, 1989

Tabela 5 - Quantidades de Unidades Domiciliares positivas para triatomíneos no peridomicilio/quantidades examinadas em cada avaliação e indices de positividade de peridomicílio.

\begin{tabular}{cccccccccc}
\hline Grupos & \multicolumn{10}{c}{ Avaliações } \\
\hline & $1 \mathrm{a}$ & $2{ }^{\mathrm{a}}$ & $3{ }^{\mathrm{a}}$ & $4{ }^{\mathrm{a}}$ & $5 \mathrm{a}$ & $6 \mathrm{a}^{\mathrm{a}}$ & $7 ?^{\mathrm{a}}$ & $8 \mathrm{a}$ & $9 \mathrm{a}$ \\
\hline $\mathrm{A}$ & $8 / 103$ & $3 / 105$ & $2 / 100$ & $4 / 105$ & $6 / 93$ & $3 / 97$ & $6 / 94$ & $7 / 93$ & $2 / 98$ \\
& $(7,8)$ & $(2,8)$ & $(2,0)$ & $(3,8)$ & $(6,4)$ & $(3,1)$ & $(6,4)$ & $(7,5)$ & $(2,0)$ \\
$\mathrm{B}$ & $5 / 83$ & $2 / 80$ & $0 / 80$ & $4 / 76$ & $4 / 82$ & $2 / 73$ & $0 / 78$ & $1 / 80$ & $1 / 76$ \\
& $(6,0)$ & $(2,5)$ & $(0,0)$ & $(5,3)$ & $(4,9)$ & $(2,6)$ & $(0,0)$ & $(1,2)$ & $(1,3)$ \\
$\mathrm{C}$ & $6 / 82$ & $0 / 85$ & $0 / 85$ & $2 / 77$ & $2 / 77$ & $3 / 71$ & $3 / 80$ & $1 / 77$ & $1 / 74$ \\
& $(7,3)$ & $(0,0)$ & $(0,0)$ & $(2,6)$ & $(2,6)$ & $(4,2)$ & $(3,8)$ & $(1,3)$ & $(1,4)$ \\
$\mathrm{D}$ & $4 / 85$ & $3 / 83$ & $2 / 87$ & $4 / 80$ & $5 / 80$ & $1 / 82$ & $0 / 84$ & $0 / 80$ & $2 / 78$ \\
& $(4,7)$ & $(3,6)$ & $(2,3)$ & $(5,0)$ & $(6,2)$ & $(1,2)$ & $(0,0)$ & $(0,0)$ & $(2,6)$ \\
\hline Sign. & NS & NS & NS & NS & NS & NS & S & S & NS \\
\hline
\end{tabular}

Das 3.043 amostras, 99 foram positivas no peridomicílio, sendo mostrado o número de amostras positivas para cada espécie, num total de 103 amostras, na Tabela 6. Fica evidente a maior quantidade de amostras positivas para T. pseudomaculata, provável indicação de melhor adaptação desta espécie nativa da região ao ambiente menos protegido pelo inseticida $\mathrm{e}$ mais exposto a reinfestação. A grande variedade de possiveis abrigos, que eram criados e destruídos com o passar do tempo, tornava dificil definir quantas U. Ds. dispunham de anexos e quais haviam sido tratados com inseticida.

Tentamos avaliar a importância do peridomicílio na reinfestação do intradomicílio, observando que nas $36 \mathrm{U}$. Ds. que foram positivas em alguma ocasião neste ambiente (Tabela 3 ), encontramos triatomineos também no peridomicilio em 12 delas; destas associações, 7 foram com a mesma espécie e 5 com espécies diferentes, sendo que em 3 dos 7 primeiros os obtivemos antes no peridomicilio.

Seria necessário um acompanhamento detalhado do peridomicilio, aparentemente muito variável na área deste estudo.

\section{DISCUSSÃO}

O nosso método de avaliação da presença de triatomíneos, usual na SUCAM, é semelhante ao usado, com bons resultados, por Pinchin e $\operatorname{col}^{10}$, que indicou pouca vantagem no uso adicional de caixas de Gomez-Nuñez.

Os índices foram muito baixos e os valores maiores dos indices cumulativos se devem à não repetição do encontro de insetos nas mesmas U. Ds.; isto indica, provavelmente, que as populaçōes eram muito pequenas e/ou de baixa persistência, pela exposição repetida às superfícies tratadas.

$T$. infestans tem sido encontrado no Brasil, com raras exceções ${ }^{2}$, somente nos domicílios. A maior quantidade de amostras positivas para esta espécie no intradomicilio deve estar ligada à sua boa adaptação aos domicilios e capacidade de proliferação a partir de pequenas populações residuais. Esta boa adaptação deve ter facilitado a dispersão e colonização em ambientes tão distantes e diferentes quanto os Andes, $o$ interior das regiões sul, sudeste e centro-oeste do Brasil e o semi-árido da Paraiba, no qual já foi encontrado em 16 municipios 6 . Fuentes e $\mathrm{col}^{4}$

Tabela 6 - Quantidades de amostras positivas no peridomicílio para cada espécie, nos grupos A, B, C e D, após a pulverização.

\begin{tabular}{crrrrr}
\hline Espécies & \multicolumn{5}{c}{ Grupos } \\
\hline & A & B & C & D & Total \\
\hline T. pseudomaculata & 34 & 14 & 18 & 19 & 85 \\
T. brasiliensis & 8 & 5 & 0 & 2 & 15 \\
T. infestans & 0 & 1 & 1 & 0 & 2 \\
P. megistus & 1 & 0 & 0 & 0 & 1 \\
\hline Total & 43 & 20 & 19 & 21 & 103 \\
\hline
\end{tabular}


Marcóndes CB, Pinto CT. Avaliação da eficiencia de deltametrina (K-Othrine 50FW), em doses baixas, no controle de triatomíneos em São Sebastião do Umbuzeiro, Paraiba. Revista da Sociedade Brasileira de Medicina Tropical 22: 85-90, Abr-Jun, 1989

obtiveram positividade em 9 de 123 casas $(7,3 \%)$, todas só com $T$. infestans, em localidades do sul de São Sebastião do Umbuzeiro e supuseram que esta espécie tende a substituir $T$. brasiliensis após a pulverização. Com a repetição de medidas profiláticas, isto parece improvável, pela disponibilidade de focos silvestres das espécies locais. A evolução da infestação por $T$. infestans, após nova pulverização geral, feita em todo o município em novembro de 1987, deve ser estudada. Em Mambai (Goiás), GarciaZapata e $\operatorname{col}^{5}$ observaram presença de T. infestans em $14,2 \%$ das U. Ds., no intradomicilio, após várias pulverizações (BHC, Malation e deltametrina), além de aumento na proporção de U. Ds. com T. sordida no peridomicílio e no intradomicílio.

A repetição das aplicações de inseticidas, acompanhada de melhoramento de habitações e educação sanitária, é muito importante para a erradicação de $T$. infestans e redução nos índices intradomiciliares de outras espécies, do mesmo modo que em Mambai. 5

T. pseudomaculata foi a segunda espécie em número de amostras positivas no intradomicilio e a primeira no peridomicilio. Estas observações são semelhantes às de Alencar ${ }^{1}$, que supõe ser esta espécie a primeira a recolonizar o intradomicilio, por se esconder no telhado. Ela parece, no entanto, ter pequena importância na transmissão de Trypanosoma cruzi ao homem, por sua acentuada ornitofilia, sendo encontrada com baixos índices de infecção ${ }^{1}$ e, experimentalmente, eliminando poucas dejeções infectantes. ${ }^{9}$

Se forem considerados os indices de invasão ou colonização, teremos proporções muito baixas de U. Ds. com triatomíneos no período. O primeiro deles não indica a formação de colônias, mas somente o encontro, no intradomicílio, dos insetos de maior probabilidade de deslocamento. Este índice depende do comportamento de vôo e do ciclo anual dos insetos, ainda desconhecido para as espécies e a região do presente estudo. Os índices de positividade também foram baixos, reforçando as indicaçōes de bom efeito do inseticida. $O$ índice cumulativo seria provavelmente rigoroso em excesso e de pouca utilidade. A não ser no caso de pulverização imediata de casas com insetos, não parece justificável, pelo menos na fase da pulverização geral, considerar definitivamente positiva e auxiliando na indicação de uma nova pulverização geral uma U. D. na qual foram obtidas, numa avaliação, alguns insetos. Estes, provavelmente, seriam eliminados pelo inseticida ou, pelo menos, teriam dificuldade na formação de colônias, como indicado pela raridade de reencontro de positividade nas $U$. Ds.

Os abrigos peridomiciliares, por sua maior exposição à invasão e colonização por triatomíneos, tratamento dificil e degradação do inseticida, têm maior tendência a ser tornarem positivos, como indicado pelos índices, em geral maiores que os do intradomicilio, deste estudo. No entanto, nota-se uma baixa associação entre positividade no intra e no peridomicilio nas mesmas U. Ds. Isto, provavelmente, indica que, na área, a infestação do peridomicílio não é muito importante como um passo na reinfestação do intradomicílio. Esta importância é pouco conhecida e a solução do problema do controle de triatomíneos neste ambiente "trata-se de verdadeiro desafio à criatividade e à capacidade de investigação" (JCP Dias: 1? Seminário sobre Vigilância Epidemiológica em Doença de Chagas, João Pessoa, 1986).

Os resultados obtidos com as quatro doses foram melhores, com doses maiores, em outras regiōes 5711 , havendo provavelmente influência das espécies envolvidas, do clima e de fatores humanos.

A ausência de vantagem evidente no uso das doses mais altas indica que a diluição de $125 \mathrm{ml} /$ bomba pode ser substituido pelas menores, com economia de até $60 \%$. Dependendo da atividade em andamento, o gasto com inseticida pode ser maior que o de diárias, atingindo 53,5 e $67,4 \%$ do custo em dois dos anos estudados em Mambai ${ }^{5}$. Seria necessário o cálculo do custo para a área do semi-árido.

A pulverização geral da área poderia ser seguida de outras, após mais de um ano, das U. Ds. positivas ou de todas. $O$ fato das $U$. Ds. positivas numa avaliação raramente estarem assim nas outras torna mais conveniente a pulverização geral da área, principalmente no caso de se pretender a erradicação de espécies perigosas como $T$. infestans. A pesquisa e erradicação de focos silvestres, como já testado em Minas Gerais (C. C. P. Loyola, dados não publicados), deveria ser analisada como medida auxiliar.

\section{SUMMARY}

We tested deltamethrin ( $K$-Othrine $50 \mathrm{FW}$ ) in the control of triatomine bugs, in an semi arid area with about $56.6 \%$ of houses bug infested. We used in 402 Domiciliary Units, $125,75,60$ or 50 ml per pump of 10 liters, equi valent, if applied on $250 \mathrm{~m}^{2}$, to 25,15 , 12 and $10 \mathrm{mg} / \mathrm{m}^{2}$. The invasion, colonization and cumulative indices in the houses and the positivity index in shelters near houses were very low and similar in the four groups during the 15 months of evaluation, indicating that lower doses could replace $25 \mathrm{mg} / \mathrm{m}^{2}$. T. infestans, followed by $T$ pseudomaculata, predominated in and near the houses, after spraying.

Key-words: Deltamethrin. Chagas disease. Prophylaxis. Semi-arid. Triatominae. 
Marcondes CB, Pinto CT. Avaliafão da eficiência de deltametrina (K-Othrine 50FW), em doses baixas, no controle de triatomíneos em São Sebastiâo do Umbuzeiro, Paraíba. Revista da Sociedade Brasileira de Medicina Tropical 22: 85-90, Abr-Jun, 1989

\section{AGRADECIMENTOS}

Aos Drs. Delosmar D. Mendonça e Judá D. Wanderley (SUCAM/PB) e J. C. P. Dias (DIDOCH/ SUCAM) e ao pessoal de campo da SUCAM, pelo inestimável apoio, ao estagiário Luiz Rogério Benvenutti e ao mestrando do NETEB/CCS/UFPb M. F. Amorim, pelo auxilio na computaçāo dos dados e à Dra. M. M. S. Nunesmaia (DE/CCEN/UFPb), pela orientação na análise estatística.

\section{REFERÊNCIAS BIBLIOGRÁFICAS}

1. Alencar JE. História natural da doença de Chagas no estado do Ceará. Imprensa Universitária, Fortaleza, 1987.

2. Barretto MP. Epidemiologia. In. Brener Z, Andrade ZA. ed Trypanosoma cruzi e doença de Chagas. p.89-151, Guanabara Koogan, Rio de Janeiro, 1979.

3. Dias JCP, Pinto CT, Barreiros H, Vasconcelos JRA, Loiola CCP, Silva MA. Emprego da deltametrina e da cyflutrina contra triatomíneos em área de Minas Gerais, Brasil Anais do 22. Congresso da Sociedade Brasileira de Medicina Tropical, página Belo Horizonte, 1986.

4. Fuentes AR, Sabroza PC, Marzochi MCA, Silva VL, Modena CM, Brito CM. Presença do Triatoma infestans (Klug, 1834) em área sujeita ao efeito residual da deltametrina no Município de São Sebastião do Umbuzeiro, Paraiba Anais da 15a Reuniaao Anual de Pesquisa Básica em Doença de Chagas (VE-29), Caxambu, 1988.

5. Garcia-Zapata MT, Marsden PD, Virgens D, Penna R, Socares V, Brasil IA, Castro CN, Prata A, Macedo V. O controle da transmissāo da doença de Chagas em
Mambai-Goiás, Brasil (1982-1984). Revista da Sociedade Brasileira de Medicina Tropical 19:219-225, 1986.

6. Marcondes CB, Mendonça D. Observações sobre a ocorrència de Triatoma infestans (Reduviidae, Triatominae) no estado da Paraiba. 10. Congresso da Sociedade Brasileira de Parasitologia p. 89, 1987.

7. Oliveira Filho AM. Field tests of 3 formulations of deltamethrin in houses colonized by Triatoma infestans. Anais da 11.a Reuniäo Anual de Pesquisa Basica em Doenca de Chagas (VE-37), Caxambu, 1984.

8. Oliveira Filho AM, Figueiredo MJ, Melo MTV, Santos CE, Silva EL. Considerations about techniques of determination of real dose sprayed in houses treated for the control of insects Anais da 5a Reunião de Pesquisa Aplicada em Doenças de Chagas, p. 52, Araxá, 1988.

9. Pereira JB, Pessoa I, Coura JR Observaçōes sobre as dejeções e o número de $T$. cruzi eliminados por diferentes espécies de triatomíneos durante a alimentação. Resultados preliminares. Anais da 15 a Reuniāo de Pesquisa Básica em Doença de Chagas (VE-20), Caxambu, 1988.

10. Pinchin R, Fanara DM, Castleton CW, Oliveira Filho AM. Comparison of techniques for detection of domestic infestations with Triatoma infestans in Brazil. Transactions of the Royal Society of Tropical Medicine and Hygiene 75:691-694, 1981.

11. Pinchin R, Oliveira Filho AM. Fanara DM, Gilbert B. Ensaio đe campo para avaliação das possibilidades de uso da decametrina (OMS 1998) no combate a triatomíneos. Revista Brasileira de Malariologia e Doenças Tropicais 32:36-41, 1980.

12. Varejâo-Silva, MA. Atlas climatológico do Estado da Paraiba. Universidade Federal da Paraiba, Campina Grande, 1984. 